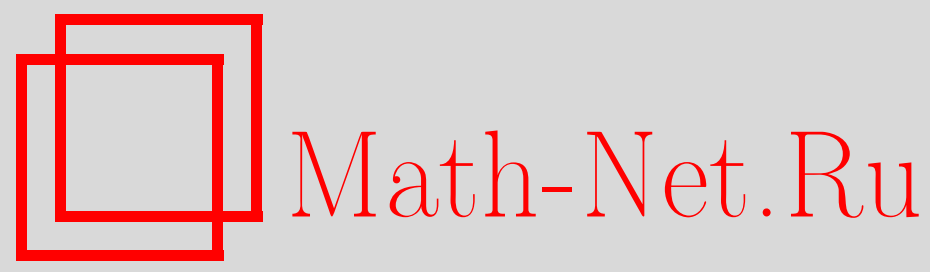

Б. Т. Батикян, С. А. Григорян, Об алгебраическом расширении $A(E)$, Матем. заметки, 2002, том 72, выпуск 5, 649 653

DOI: https://doi.org/10.4213/mzm452

Использование Общероссийского математического портала Math-Net.Ru подразумевает, что вы прочитали и согласны с пользовательским соглашением http://www.mathnet.ru/rus/agreement

Параметры загрузки:

IP : 35.173 .219 .149

26 апреля 2023 г., 06:03:42

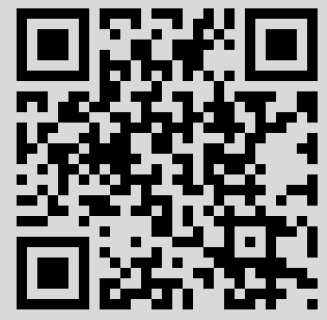


УДК 517.986

\title{
ОБ АЛГЕБРАИЧЕСКОМ РАСШИРЕНИИ $A(E)$
}

\author{
Б. Т. Батикян, С. А. Григорян
}

Алгебраическое расширение алгебры $A(E)$, где $E$ - компакт в $\mathbb{C}$ с непустой связной внутренностью, приводит к банаховой алгебре $B$ функций, голоморфных на некотором аналитическом множестве $K^{\circ} \subset \mathbb{C}^{2}$ с краем $b K$ и непрерьвных вплоть до $b K$. Изучаются особые точки спектра алгебры $B$ и их дефекты. В случае, когда $B$-равномерная алгебра, оценивается глубина $B$ в алгебре $C(b K)$. В частности, получены условия, обеспечивающие максимальность $B$ на $b K$.

Библиография: 11 названий.

Пусть $E$ - компактное подмножество комплексной плоскости $\mathbb{C}$ с непустой и связной внутренностью $E^{\circ}, A=A(E)$ - равномерная алгебра всех непрерывных на $E$ и голоморфных в $E^{\circ}$ функций, и пусть $A[t]$ - алгебра всех полиномов над $A$. Рассмотрим унитарный полином

$$
p=t^{n}+a_{1} t^{n-1}+\cdots+a_{n}
$$

из $A[t]$ и обозначим через $B$ алгебраическое расширение алгебры $A$, порожденное полиномом $p$, т.е. положим $B=A[t] /(p)$, где $(p)$ - главньй идеал в $A[t]$, образованньй $p$. (Подробнее об алгебраическом расширении, назьваемом также расширением АренсаГофмана, коммутативной банаховой алгебры см. [1], [2].)

Мы будем предполагать, что дискриминант $d$ полинома $p$ есть ненулевой элемент алгебры $A$, и, не ограничивая общности, что коэффициенты $p$ подчинены условию $\sum\left\|a_{i}\right\| \leqslant 1$.

Расширение $B$ есть полупростая коммутативная банахова алгебра относительно нормы

$$
\left\|\sum_{k=0}^{n-1} f_{k} t^{k}\right\|=\sum_{k=0}^{n-1}\left\|f_{k}\right\|, \quad f_{k} \in A,
$$

причем алгебра $A$ изометрически и изоморфно вкладывается в $B$. Спектром (пространством максимальных идеалов) для алгебры $B$ служит компакт

$$
K=\left\{(z, \lambda) \in E \times \mathbb{C}: P(z, \lambda)=\lambda^{n}+\lambda^{n-1} a_{1}(z)+\cdots+a_{n}(z)=0\right\},
$$

а естественное отображение $\pi: K \rightarrow E$ спектров алгебр $B$ и $A$, вызванное вложением $A$ в $B$, есть $n$-листное и, вообще говоря, разветвленное накрытие, голоморфное над $E^{\circ}$. Известно также, что граница Шилова $\partial B$ алгебры $B$ совпадает с прообразом $\pi^{-1}(b E)$ топологической границы $b E(=\partial A)$ компакта $E$. 
Для удобства прообраз $\pi^{-1}(b E)$ мы будем обозначать через $b K$, а через $K^{\circ}-$ прообраз внутренности компакта $E$.

Функцию $g$ называют $B$-голоморфной в точке $x \in K$, если имеется такая открытая окрестность $V \subset K$ точки $x$, что $g$ равномерно на $\bar{V}$ приближается элементами алгебры $B$. Если $x$ не является точкой ветвления накрытия $\pi$, т.е. $d(\pi(x)) \neq 0$, то эквивалентное определение заключается в существовании такой окрестности $\bar{V}$, гомеоморфной $\pi(\bar{V})$, что функция $\pi^{-1} \circ g$ принадлежит $A(\pi(\bar{V}))$.

Приведем применительно к рассматриваемой ситуации определения особой точки спектра полупростой коммутативной банаховой алгебры и дефекта такой точки,предложенные в нашей работе [3]. Пусть $x \in K, \mathscr{O}_{B}(x)$ - алгебра ростков всех $B$-голоморфных в окрестности $x$ функций, $\mathscr{O}_{B}^{c}(x)$ (соответственно $\left.\mathscr{O}_{B}^{*}(x)\right)$ - алгебра ростков всех тех функций, которые непрерьвны (соответственно ограничены) в окрестности $x$ и $B$-голоморфны в ее проколотой окрестности. Положим

$$
m_{B}(x)=\operatorname{dim} \mathscr{O}_{B}^{c}(x) / \mathscr{O}_{B}(x)
$$

$($ кратность точки $x)$ и

$$
r_{B}(x)=\operatorname{dim} \mathscr{O}_{B}^{*}(x) / \mathscr{O}_{B}^{c}(x)
$$

(порядок приводимости $x$ ). Дефектом точки $x$ назьвается число

$$
\delta_{B}(x)=m_{B}(x)+r_{B}(x)
$$

а точка $x$ спектра алгебры $B$ считается особой, если $\delta_{B}(x)>0$.

Например, всякая неизолированная точка $b K$ является особой, так как согласно теореме 4.3 из [3] дефект такой точки бесконечен.

Пусть теперь $x \in K^{\circ}$. Понятно, что $K^{\circ}$ - одномерное аналитическое множество в $\mathbb{C}^{2}$, а пучок ростков $\left\{\mathscr{O}_{B}(x)\right\}_{x \in K^{\circ}}$ является структурным пучком для $K^{\circ}$. Поскольку $\mathscr{O}_{B}^{*}(x)$ совпадает с целым замыканием локальной алгебры $\mathscr{O}_{B}(x)$, то из когерентности структурного пучка следует:

1) конечность дефекта $\delta_{B}(x)$ для всякого $x$ из $K^{\circ}$;

2) эквивалентность приведенного выше определения особой точки обычному определению особой точки одномерного аналитического множества.

Отметим также, что если $K^{\circ}$ - алгебраическое множество, т.е. если коэффициенты полинома $p$ сами являются полиномами от $z$, то дефект точки $x \in K^{\circ}$ равен введенному Серром числу $\delta_{x}$ (см. $[4$, с. 84$\left.]\right)$.

В том случае, когда аналитическоемножество $K^{\circ}$ неприводимо, дефект точки эффективно описьвается формулой Милнора

$$
2 \delta_{B}(x)=\mu(x)+r_{B}(x)
$$

где $\mu(x)$ - число Милнора точки $x$, т.е. логарифмический вычет в $x$ градиента голоморфной функции $P(z, \lambda)[5$, c. 91$]$. Если точка $x$ неприводима, то $2 \delta_{B}(x)=\mu(x)$, если же $r_{B}(x)>0$, то

$$
2 \delta_{B}(x) \geqslant\left(r_{B}(x)+1\right) r_{B}(x) .
$$


ЗАмЕчАниЕ. Последнее неравенство означает, что при алгебраическом расширении алгебры аналитических функций кратность $m_{B}(x)$ и порядок приводимости $r_{B}(x)$ точки спектра расширения подчинены определенным соотношениям. В общем случае, как показывают теоремы 3.1 и 4.1 из [3], эти числа могут быть совершенно произвольными.

Заметим также, что множество $\operatorname{Sing}\left(K^{\circ}\right)$ особых точек $K^{\circ}$ дискретно и содержится (вообще говоря, строго) во множестве $\pi^{-1}\left(N_{d} \cap E^{\circ}\right)$ точек ветвления накрытия $\pi \mid K^{\circ}$, где $N_{d}$ - множество нулей дискриминанта $d$.

Предполагая конечность множества $\operatorname{Sing}\left(K^{\circ}\right)$, рассмотрим следующие равномерные алгебры: $\mathfrak{B}$ - всех $B$-голоморфных функций на $K$, a $\mathfrak{A}$ - всех ограниченных и $B$-голоморфных функций на

$$
K^{*}=K \backslash \operatorname{Sing}\left(K^{\circ}\right) \text {. }
$$

Очевидно, $\mathfrak{B} \subset \mathfrak{A}$ и, как нетрудно убедиться, $\partial \mathfrak{A}=\partial \mathfrak{B}=b K$.

TеОрема 1. Если множество $\operatorname{Sing}\left(K^{\circ}\right)$ содержит конечное число точек $x_{1}$, $\ldots, x_{m}, m o$

$$
\operatorname{dim} \mathfrak{A} / \mathfrak{B}=\sum_{i=1}^{m} \delta_{B}\left(x_{i}\right)
$$

ДокАЗАтЕЛЬСтво. Рассмотрим на компакте $K$ пучок $\mathscr{O}=\left\{\mathscr{O}_{B}(x)\right\}_{x \in K}$ ростков $B$-голоморфных функций и пучок $\mathscr{O}^{\prime}=\left\{\mathscr{O}^{\prime}(x)\right\}_{x \in K}$, где $\mathscr{O}^{\prime}(x)=\mathscr{O}_{B}(x)$, если $x \neq x_{i}$, и $\mathscr{O}^{\prime}(x)=\mathscr{O}_{B}^{*}(x)$, если $x=x_{i}$. Понятно, что $\mathscr{O}$ есть подпучок $\mathscr{O}^{\prime}$. Точная последовательность пучков

$$
0 \rightarrow \mathscr{O} \rightarrow \mathscr{O}^{\prime} \rightarrow \mathscr{O}^{\prime} / \mathscr{O} \rightarrow 0
$$

порождает точную последовательность

$$
0 \rightarrow H^{0}(K, \mathscr{O}) \rightarrow H^{0}\left(K, \mathscr{O}^{\prime}\right) \rightarrow H^{0}\left(K, \mathscr{O}^{\prime} / \mathscr{O}\right) \rightarrow H^{1}(K, \mathscr{O})
$$

групп когомологий компакта $K$ с коэффициентами в этих пучках. Нетрудно убедиться, что алгебры сечений $H^{0}(K, \mathscr{O})$ и $H^{0}\left(K, \mathscr{O}^{\prime}\right)$ совпадают соответственно с алгебрами $\mathfrak{B}$ и $\mathfrak{A}$, а пучок $\mathscr{O}^{\prime} / \mathscr{O}$ сосредоточен на конечном множестве $\operatorname{Sing}\left(K^{\circ}\right)$ и

$$
\operatorname{dim} H^{0}\left(K, \mathscr{O}^{\prime} / \mathscr{O}\right)=\sum_{i=1}^{m} \delta_{B}\left(x_{i}\right)
$$

Учитьвая тривиальность группы $H^{1}(K, \mathscr{O})$ (см. [6, теорема 3.1]), получаем требуемое равенство.

Прежде чем перейти к следующей теореме, отметим, что спектр $S(\mathfrak{B})$ алгебры $\mathfrak{B}$ совпадает с $K[7]$ и что согласно теореме 1 естественная проекция спектров $\rho: S(\mathfrak{A}) \rightarrow K$ биективна над $K^{*}$.

TEOPEMA 2. Ecлu

1) мнохсество точек пика алгебры $A$ совпадает с bE,

2) дискриминант $d$ не обращается в нуль на $b E$,

3) аналитическое множсество $K^{\circ}$ неприводимо, то алгебра $\mathfrak{A}$ есть максимальная подалгебра алгебры $C(b K)$. 
ДокАЗАТЕЛЬСтво. Отметим предварительно, что из условия 2) теоремы следует эквивалентность нормы (1) sup-норме на $K$ [8], а тогда условие 3) обеспечит, как легко видеть, совпадение алгебр $B$ и $\mathfrak{B}$.

Пусть $\mathfrak{D}$ - некоторая равномерная алгебра на $b K$, содержащая $\mathfrak{A}$, и $\sigma: S(\mathfrak{D}) \rightarrow S(\mathfrak{A})-$ соответствующая проекция спектров. Положим $\tau=\sigma \circ \rho$ и обозначим через $F$ образ отображения $\tau$ в $K$. Ясно, что $b K \subset F$.

Предположим, что $b F$ содержит регулярную точку $x$ из $K^{\circ} \cap K^{*}$. Тогда найдутся такие замкнутая окрестность $\bar{V}$ точки $x$ и функция $g \in B_{\bar{V}}$, что $\bar{V} \cap b K=\varnothing$, а $g$ биголоморфно отображает $\bar{V}$ на замкнутый диск. В частности, $\tau^{-1}(\bar{V})$ есть $\mathfrak{D}$-вьпуклый компакт в $S(\mathfrak{D})$, а функция $h=\tau \circ g$ принадлежит $\mathfrak{D}_{\tau^{-1}(\bar{V})}$. Так как $x \in b F$, то

$$
g(x) \in b g(F \cap \bar{V}) \subset h\left(\partial \mathfrak{D}_{\tau^{-1}(\bar{V})}\right) .
$$

Но согласно локальному принципу Росси

$$
\partial \mathfrak{D}_{\tau^{-1}(\bar{V})} \subset \tau^{-1}(b \bar{V}),
$$

т.е. $g(x) \in g(b \bar{V})$, что невозможно.

Таким образом,

$$
b F \subset b K \cup \operatorname{Sing}\left(K^{\circ}\right) .
$$

Но тогда в силу неприводимости $K^{\circ}$ либо

$$
F \subset b K \cup \operatorname{Sing}\left(K^{\circ}\right),
$$

либо $K^{*} \subset F$. Далее, из условия 1$)$ следует, что каждая точка $b K$ есть точка пика для $\mathfrak{B}$, и, значит, отображение $\tau$ инъективно над $b K$.

a) Пусть $F \subset b K \cup \operatorname{Sing}\left(K^{\circ}\right)$. Так как $b K \subset F$, то по теореме Шилова об идемпотентах $S(\mathfrak{D})=b K$. Но тогда согласно теореме 3 из [9] $\mathfrak{D}$ содержит алгебру $C(b E)$. В частности, максимальные множества антисимметрии алгебры $\mathfrak{D}$ должны содержаться в слоях

$$
(\tau \circ \pi)^{-1}(z), \quad z \in b E .
$$

Поскольку все эти слои конечны, согласно теореме Шилова-Бишопа $\mathfrak{D}=C(b K)$.

б) Пусть $F=K$. Заметим, что функция $\tau \circ \pi$ принадлежит алгебре $\mathfrak{D}$, а слои

$$
(\tau \circ \pi)^{-1}(z), \quad z \in b E
$$

содержат не более $n$ точек. Согласно теореме Сеничкина (см. [10, теорема 4]) для любого $z \in E^{\circ}$ слой $(\tau \circ \pi)^{-1}(z)$ также содержит не более $n$ точек. В частности, отображение $\sigma$ биективно над $K^{*}$, и элементы алгебры $\mathfrak{D}$ можно рассматривать как ограниченные непрерьвные функции на $K^{*}$. Если $x \in K^{*} \cap K^{\circ}$, то найдется такая окрестность $\bar{V}$ точки $x$, которая биголоморфна диску, а $\mathfrak{A}_{\bar{V}}$ изоморфна диск-алгебре. Так как $S(\mathfrak{D})=\bar{V}$ и $\partial \mathfrak{D}_{\bar{V}}=b \bar{V}$, то по теореме Вермера о максимальности $\mathfrak{A}_{\bar{V}}=\mathfrak{D}_{\bar{V}}$. Если, наконец, $x \in b K^{\circ}$, то выберем такой открытый диск $\Delta$ с центром в $\pi(x)$, что $\bar{\Delta} \cap E$ гомеоморфно $\pi^{-1}(\bar{\Delta} \cap E)$, и обозначим через $U$ ту связную компоненту $\Delta \cap E^{\circ}$, замыкание которой содержит $\pi(x)$. Положим $\bar{V}=\pi^{-1}(\bar{U})$ и заметим, что $\mathfrak{A}_{\bar{V}}$ изоморфна алгебре $A(\bar{U})$, поскольку $A(\bar{U})=A_{\bar{U}}$. Но в силу условия 1$)$ алгебра $A(\bar{U})$ максимальна 
на $b \bar{U}$ (см., например, [10]), что так же, как и выше, приводит к равенству $\mathfrak{D}_{\bar{V}}=\mathfrak{A}_{\bar{V}}$. Итак, в случае б) $\mathfrak{D}=\mathfrak{A}$, и теорема доказана.

Теоремы 1 и 2 позволяют описать тип алгебраического расширения алгебры $A(E)$. Напомним, что типом $t(B)$ равномерной алгебры $B$ назьвается верхняя грань длин цепочек равномерных алгебр, соединяющих $B$ c $C(b K)$. Максимальная алгебра суть алгебра типа 1, а тип подалгебры конечной коразмерности $k$ максимальной алгебры равен $k+1$ (см. [11]).

СлЕДСТВИЕ. В условиях теоремы 2 равномерная алгебра В является алгеброй конечного типа на $b K$, причем

$$
t(B)=\sum_{x \in \operatorname{Sing}\left(K^{\circ}\right)} \delta_{B}(x)+1 .
$$

В частности, если $K^{\circ}$ - неособое аналитическое множество, то В максимальна ${ }_{\boldsymbol{B}} C(b K)$.

ЗАмЕчАниЕ. Если условие 2) теоремы заменить более слабым предположением конечности множества $\operatorname{Sing}\left(K^{\circ}\right)$, то повторяя доказательство, мы установим максимальность на $b K$ алгебры $\mathfrak{A}^{*}$ всех тех непрерывных ограниченных функций на $K^{*}$, которые $B$-голоморфны на $K^{\circ} \backslash \operatorname{Sing}\left(K^{\circ}\right)$.

\section{СПИСОК ЦИТИРОВАННОЙ ЛИТЕРАТУРЫ}

[1] Arens R., Hoffman K. Algebraic extensions of normed algebras // Proc. Amer. Math. Soc. 1956. V. 7. P. 203-210.

[2] Lindberg J.A. Algebraic extensions of commutative Banach algebras // Pacific J. Math. 1964. V. 14. P. 559-583.

[3] Batikyan B.T., Grigorian S.A. Singular points of spectra of uniform algebras // J. Cont. Math. An. 1998. V. 33. № 1. P. 1-16.

[4] Серр Ж. Алгебраические группы и поля классов. М.: Мир, 1968.

[5] Милнор Дж. Особые точки комплексных гиперповерхностей. М.: Мир, 1971.

[6] Rickart C. E. Analytic functions of an infinite numbers of complex variables // Duke Math. J. 1969. V. 36. P. 581-597.

[7] Rickart C. E. The maximal ideal space of functions locally approximable in a function algebra // Proc. Amer. Math. Soc. 1966. V. 17. P. 1320-1326.

[8] Heuer G. A., Lindberg J. A. Algebraic extensions of continuous function algebra // Proc. Amer. Math. Soc. 1963. V. 14. P. 337-342.

[9] Батиклн Б. Т., Григорян С. А. О равномерных алгебрах, содержащих $A(K) / /$ Изв. АН АрмССР. Матем. 1989. Т. 24. № 6. С. 547-556.

[10] Сеничкин В.Н. Субгармонические функции и аналитическая структура в пространстве максимальных идеалов равномерной алгебры // Матем. сб. 1979. Т. 108. № 1. С. 115-133.

[11] Батикян Б. Т., Григорян С. А. О функциональных алгебрах конечного типа // УМН. 1974. T. 29. №6. C. $155-166$.

(Б. Т. Батикян) Институт математики АН Армении, г. Ереван

(С. А. Григорян) Казанский государственный университет

Поступило

E-mail: bag@instmath.sci.am, Suren.Grigorian@ksu.ru

09.02.1999

Исправленньй вариант 12.02 .2002 